\title{
Numerical simulation of the turbulence effect on heat transfer between fluid and thin plates of a solid material
}

\author{
Nikolay Kozyulin ${ }^{1,}{ }^{*}$, Maxim Bobrov ${ }^{1}$, and Michael Hrebtov ${ }^{1}$ \\ ${ }^{1}$ Institute of Thermophysics of SB RAS, 630090 Ac. Lavrentiev ave. 1, Novosibirsk, Russia
}

\begin{abstract}
We present the results of numerical simulation of conjugate heat transfer between thin $(100-1000 \mathrm{~nm})$ solid plates and periodically heated air flow. Several air inflow conditions were studied with different turbulence intensity and integral scale. The thermal response in the plate was calculated showing that inflow turbulence interacting with the plate generates significant temperature fluctuations in the plate volume. These fluctuations amplitudes grow along the plate and with time for several periods, indicating the existence of some feedback process, with turbulent air flow acting as a medium, transferring thermal signals between different sections of the plate. The described spatial and temporal fluctuations may be used for pyroelectric energy harvesting applications.
\end{abstract}

\section{Introduction}

Heat transfer in the boundary layer has great practical importance for many engineering applications. One such application is the conversion of thermal energy into electricity using the pyroelectric effect. In such converters, thin films or plates of ferroelectrics are used with small thin electrodes deposited on their surface. These devices could be installed inside the hot exhaust pipes of automobiles or in gas/diesel electric generators to increase their efficiency.

The hot gas or liquid flowing onto the solid plates can be sufficiently turbulent. In that case, turbulence can affect heat exchange with the wall. There are some works [1,2] investigating the influence of turbulence on the spatially developing mixing layer, but their main focus is to investigate the influence of turbulence on hydrodynamics. The thermal effect is especially interesting if the incoming fluid temperature is alternating in time and the plate thickness is small. The longitudinal roll-instabilities in the boundary layer arising from the interaction of turbulent flow with the wall may affect the heat transfer locally, thus leading to the local increase of temperature oscillations amplitude. This effect in principle could be utilized in pyroelectric harvesting.

The purpose of this work was to investigate the effect of inflow turbulence on the heating and cooling of an array of thin $(100 \mathrm{~nm}-1000 \mathrm{~nm})$ ferroelectric ceramic plates by an air flow using direct numerical simulation of the conjugate heat transfer process.

*Corresponding author: nikkozyulin@gmail.com 


\section{Computational details}

The single "cell" of the plate-grid was simulated. The computational domain consists of one plate, inlet and outlet sections (Fig. 1a). The computational domain dimensions are 20 $\mathrm{mm} \times 2 \mathrm{~mm} \times 6 \mathrm{~mm}$ in longitudinal, vertical and transversal directions respectively. The curvilinear grid of $4.4 \times 10^{6}$ hexagonal cells was constructed with refinement in the nearwall region $\left(\Delta y_{0}^{+}=0.15\right)$. The symmetry conditions were set at the top and bottom boundaries and periodic conditions in the direction transversal to the flow. At the inlet a uniform velocity, $\boldsymbol{U}$, profile of $15 \mathrm{~m} / \mathrm{s}$ was superimposed with turbulent fluctuations obtained from separate simulation of isotropic turbulence. The turbulence integral scale was varied between 0.05 and $0.5 \mathrm{~mm}$. The inlet turbulence intensity $\left(E_{\text {turb }} / \boldsymbol{U}^{2}\right)$ was set to be $6 \%$. The temperature field at the inlet was set uniform and alternating in time to simulate fast changes between two temperature values ( $273 \mathrm{~K}$ and $373 \mathrm{~K})$ as follows:

$$
T_{\text {in }}(t)=323+50 \cdot \operatorname{sign}(\sin (2 \pi \omega t))|\sin (2 \pi \omega t)|^{0.1},
$$

where $\omega=500 \mathrm{~Hz}$ is the temperature alternation frequency.
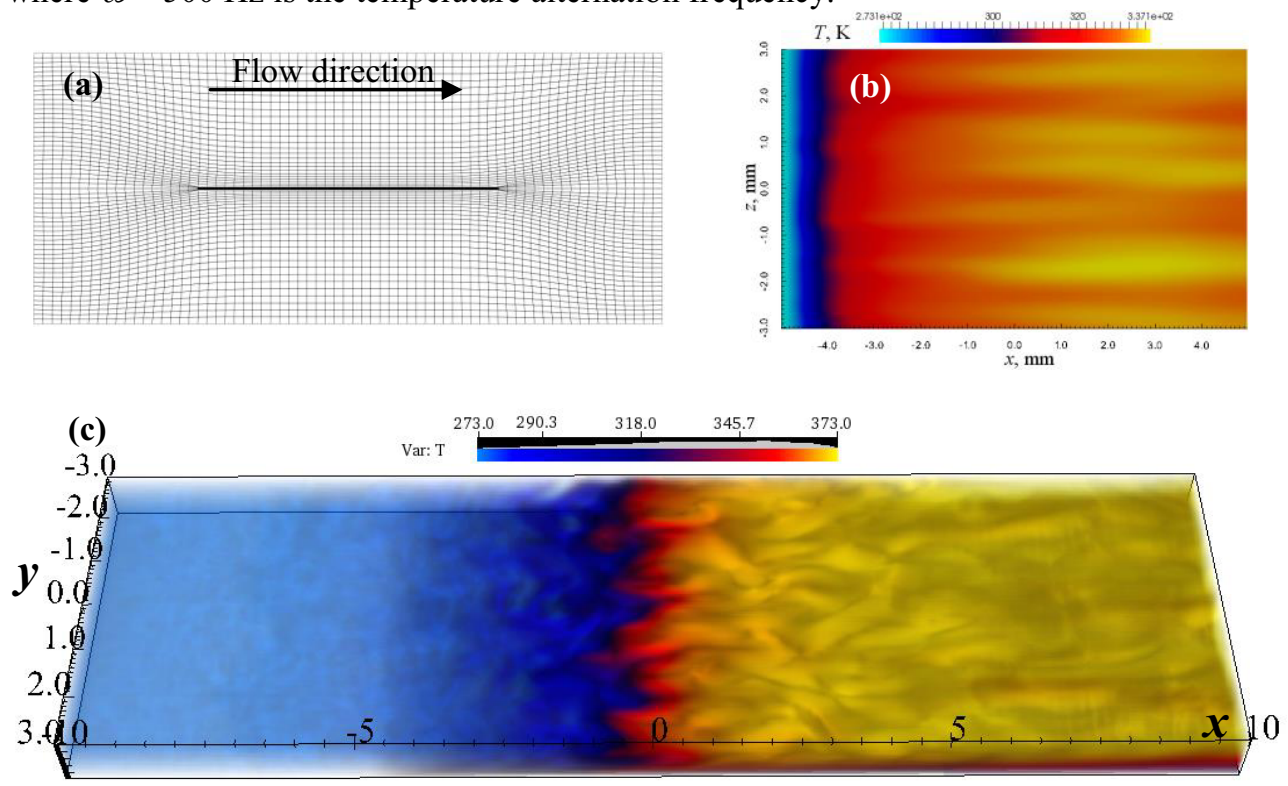

Fig. 1. The schematic of computational domain and mesh, not to scale (a); the temperature distribution at the solid plate for one moment in time (b); the temperature distribution in the fluid with the cool temperature front passing the centre of the domain (c).

The plate thickness was varied between $100 \mathrm{~nm}$ and $1000 \mathrm{~nm}$. The plate material was chosen to be barium titanate, which physical properties were taken from [3]. The fluid was chosen to be air (density $\rho=1 \mathrm{~kg} / \mathrm{m}^{3}$, specific heat $\mathrm{c}_{\mathrm{p}}=1 \mathrm{~kJ} /(\mathrm{kg} \mathrm{K})$, thermal conductivity $K$ $=0.03 \mathrm{~W} /(\mathrm{mK})$, and kinematic viscosity $\left.v=2.1 \times 10^{-5} \mathrm{~m}^{2} / \mathrm{s}\right)$. The temperature dependencies of viscosity and thermal conductivity were neglected at present simulations.

The simulations were done with modified conjugate heat transfer solver from OpenFoam [4] package, second order accurate in space and time. Due to low Reynolds number of the flow (Re 1500, based on the height of computational domain) the Kolmogorov scale is resolved at the chosen grid and the simulation could be considered as 
DNS, with no subgrid model used. The preliminary computations on the sequence of refined grids showed that the current setup is sufficient for the flow considered.

\section{Results and discussion}
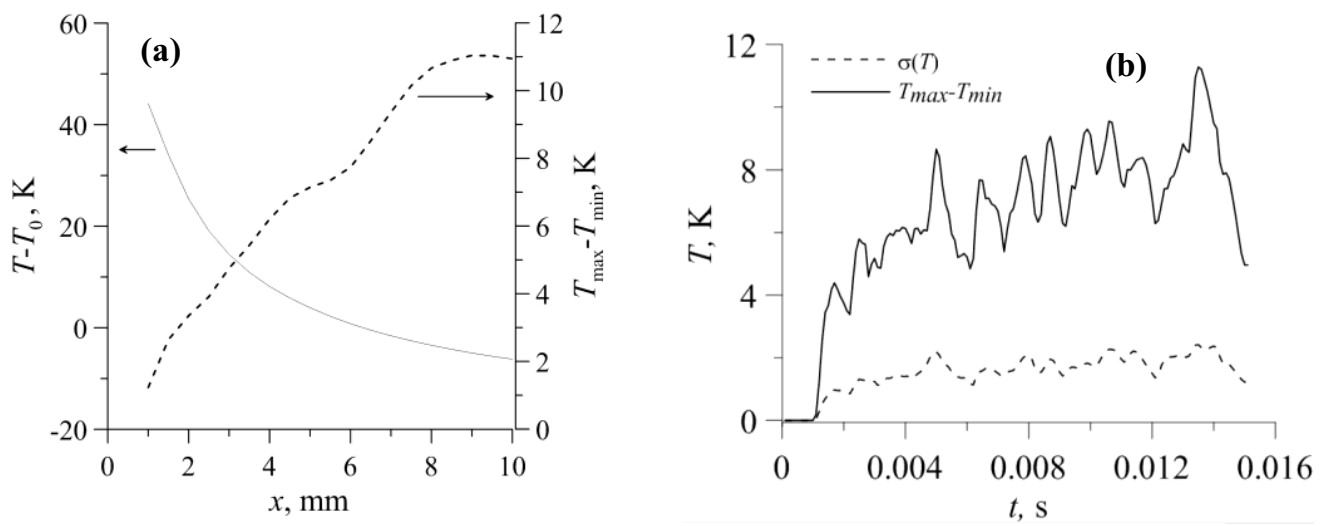

(c)
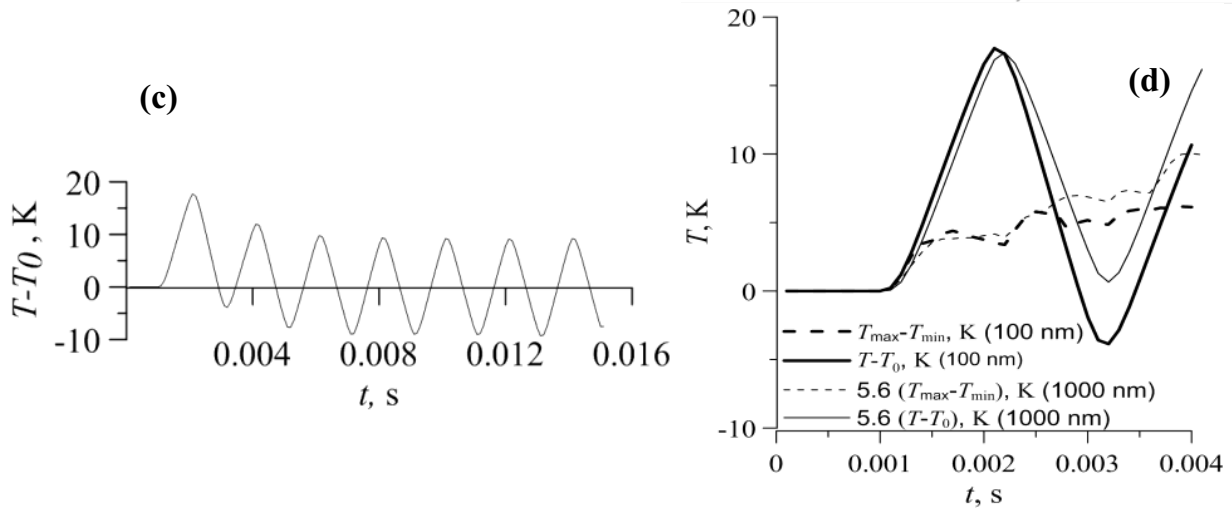

Fig. 2. Plate temperature distribution (averaged over transversal direction) along central longitudinal cross-section together with temperature fluctuations amplitude (at $t=0.0115 \mathrm{~s})(\mathrm{a})$; temperature fluctuations time evolution at the end of the plate $(x=9 \mathrm{~mm})(\mathrm{b})$; temperature evolution at $x=9 \mathrm{~mm}$ (c); Temperature and thermal fluctuations evolution for different plate thicknesses (d). .

The effect of turbulent vortices moving with the hot/cold temperature front above the solid surface leads to the formation of longitudinal stripes of hot/cold temperature at the surface (Fig 1b). These stripes are generated due to three-dimensional boundary layer instabilities triggered by inflow turbulence. The longitudinal roll-vortices which are the dominant mode of the instability intensify the heat transfer at some places and at some other places diminish it. The computation of evolution of the 100 -nm-thick plate temperature averaged in transversal direction showed that turbulence has very weak effect on the averaged temperature. The mean temperature evolution for 8 cycles was the same with and without inflow turbulence giving only about $0.5 \%$ difference at most. However temperature fluctuations inside the plate generated along the transversal direction by the interaction with turbulent flow are quite significant.

We used the difference between the highest and lowest temperature along the transversal cross-sections together with the standard deviation of temperature to measure the intensity of temperature variations.

Fig 2a showing the temperature distribution in central longitudinal cross-section of a 100 -nm-thick plate in the first half of seventh heating cycle. It is seen that the mean 
temperature at the beginning of the plate is much higher that at the plate's end. This is partially due to the loss of heat by the air when it moves along the cooler plate and partially due to the growth of boundary layer thickens. However as it can be seen the temperature fluctuations in the transversal direction are the highest at the plate's end and their magnitude is comparable with the fluctuations caused by the prescribed temperature alteration at the same point (see Fig 2c). What is more interesting, the fluctuations' magnitude at the plate's end is growing with time steadily for at least 6 periods (Fig2 b). This may be explained by some thermal feedback effect of the fluctuations at the plate's beginning on the plate's end. Looking at the evolution of two transversal cross-sections: one at the beginning of the plate and the other at the plate's end it is clearly seen that during the first few cycles the local extrema of the temperature fluctuations are uncorrelated between the cross-sections but then they become more and more correlated (temperature minimums at one cross-section starts to coincide with maximums at the other) and simultaneously the magnitude of the fluctuations at the plate's end starts to grow. This effect needs further investigation and here we only outline our current findings.

The effect of plate thickness on the fluctuations magnitude was also investigated. Fig. $2 \mathrm{~d}$ shows the normalized mean temperature and fluctuations evolution during the first cycle of simulation for two different plate thicknesses $(100 \mathrm{~nm}$ and $1000 \mathrm{~nm})$. It could be seen that relative magnitude of fluctuations in comparison with the mean temperature is roughly the same that means that the effect might be reproduced to some extent on the thicker plates. The separate study is needed to find the most effective setup for the generation of strong temperature fluctuations.

Our simulations showed that the integral scale of turbulence is an important parameter for the strong temperature fluctuations to take place, the integral scale should be comparable with the boundary layer rolls diameter (in our case $\sim 0.5 \mathrm{~mm}$ ) smaller integral scales give much weaker fluctuations $(0.25 \mathrm{~mm}$ integral scale gives $\sim 4$ times less fluctuations magnitude).

\section{Conclusion}

The described effect of thermal fluctuations generation by the interaction of inflow turbulence with the spatially growing boundary layer conceptually may be used to improve the efficiency of pyroelectric harvesting. The hot/cold temperature "stripes" caused by the roll-vortices are predominantly longitudinal. This allows one to use separate longitudinal electrodes on the surface of ferroelectric material to harvest the fluctuations energy. The width of the electrodes should be the same as the roll-vortices diameter, which could be easily calculated for each situation. Further research is needed to find the optimal parameters for the generation of the strongest fluctuations.

This work was supported by the Russian Science Foundation (grant №16-19-00119).

\section{References}

1. T.S. Lund, X. Wu, K.D. Squires, J. Comput. Phys. 140, 233 (1998)

2. A. Ferrante, S.E. Elghobashi, J. Comput. Phys. 198, 372 (2004)

3. Y. He, Thermochim. Acta. 419, 135 (2004)

4. H.G. Weller, G. Tabor, H. Jasak, C. Fureby, Computers in Phys. 12, 620 (1998) 